\title{
Study on Phenotypic, Genotypic Correlation and Path Coefficients in Chilli
}

\author{
T.S. Mishra ${ }^{1}$ and H.M. Singh ${ }^{2 *}$ \\ ${ }^{1}$ Krishi Vigyan Kendra West Kameng, Dirang Arunachal Pradesh, India \\ ${ }^{2}$ National Horticultural Research and Development Foundation, Patna (Bihar), India \\ *Corresponding author
}

\section{A B S T R A C T}

\begin{tabular}{l} 
Key w o r d s \\
Chilli, Phenotypic \\
correlation, \\
Genotypic \\
correlation and path \\
coefficients \\
\hline Article Info \\
$\begin{array}{l}\text { Accepted: } \\
\text { 17 June } 2020 \\
\text { Available Online: } \\
\text { 10 July } 2020\end{array}$ \\
\hline
\end{tabular}

\section{Introduction}

Chilli (Capsicum annuum) is one of the most important vegetable as well as spice crop, belongs to family solanaceae. It is a self pollinated crop bearing a pod like fruit (berry) and has a predominant position among the spices grown all over India. The information usually needed for developing high yielding varieties in a particular species pertains to the extent of genetic variability for desirable traits in the available germplasm. Large variability ensures better chances of producing new forms. The Phenotypic variability includes both genotypic and environmental variation and hence change under different environmental conditions. Genotypic variability remains unchanged by environmental condition.

Correlation coefficient is the mutual liner relationship between two or more variables. Correlation coefficient among different characters is either positive or negative and it 
may be high or low. Estimation of correlation coefficient among the yield contributing characters is necessary to understand the direction of selection and to maximize yield in the shortest period of time. Path coefficient provides an effective means of entangling direct and indirect causes of association of selection and measures the relative importance of each causal factor. chilli being an important crop in Uttar Pradesh needs attention about genetic improvement.

Therefore, keeping the above factors in view the present investigation was undertaken to find out the following objectives, the study of phenotypic correlation, genotypic correlation and path coefficients between characters.

\section{Materials and Methods}

The present investigation include a brief description of the site of the experiment, soil properties, climatic conditions prevalent in the locality during the period of experiment, statistical analysis, particulars of treatment, planting materials used and sampling techniques. A field experiment was conducted at Horticulture Research Farm (HRF), Department of Horticulture, Allahabad (U.P). The different variety was taken for the study from IIVR Varanasi Varieties Pusa Jawala, SM-20, JCA-9, LCA-206, LCA-404, IC413702, Japani long, Pbc-1438, IC- 383079, LCA-333,LCA-334, CO-5686-1, Kashi Anmol, LCA-357, EC-492576, Utkal Ragini, LCA-324, LCA-312, LCA-301, LCA-304.

The phenotypic variance, genotypic variance and Environmental variance (Components of variance) was calculated by subtracting the mean squre at treatment level as proposed by Burton and De Vane (1953). The phenotypic and genotypic correlation coefficients were worked out to study the inter-relationship between various pairs of characters as suggested by AL-Jibouri et al., (1958). The path coefficient were obtained according to the procedure suggested by Dewey and Lu (1959) using phenotypic and genotypic correlation coefficients. The direct and indirect effects are rated as follows by Lenka and Mishra (1973).

\section{Results and Discussion}

\section{Phenotypic correlation coefficient}

The correlation coefficient at phenotypic and genotypic levels was computed among 17 characters for 20 genotypes and their correlation with level of significance is given in table $1 \&$ table.2. A perusal of correlation coefficients revealed that genotypic correlation coefficients were in general higher than the corresponding phenotypic correlation due to Rabi season crop. At the phenotypic levels fruit yield showed positive and significant association with green fruit yield. The plant height per plant (100 and 120 days) showed significant positive phenotypic correlation with average green fruit yield per plant. Days to 50 percent flowering, days to $1^{\text {st }}$ flowering and days to green fruit diameter showed highly significant negative correlation with the green fruit and red ripe fruit yield. Average weight of green fruit showed highly significant positive correlation with phenotypic correlation with green fruit yield and red ripe fruit yield. Average fruit length and average fruit diameter showed significant positive phenotypic correlation with green fruit yield and red ripe fruit yield. Days to $1^{\text {st }}$ ripe fruit harvest showed significant negative phenotypic correlation with green fruit yield. Average weight of red ripe and average, red ripe fruit yield showed significant positive correlation with phenotypic correlation with green fruit yield. Number of seeds/plant and weight of seeds/fruit showed significant positive phenotypic correlation with green fruit and red yield at $1 \%$ level at significance. Ascorbic acid, oleoresin and capsaicin content 
showed significant positive phenotypic correlation with green fruit and red ripe fruit yield at $1 \%$ level of significant whereas, capsaicin content was found significant at $5 \%$ level of significance.

\section{Genotypic correlation coefficient}

Plant height for plant (100 and 120 days) showed highly significant positive genotypic correlation with green and red ripe fruit yield. Days to first flowering and days to $50 \%$ flowering showed highly significant positive and negative genotypic correlation with green fruit yield and red ripe fruit yield. Days to first green fruit harvest and days to first ripe fruit harvest highly significant negatively correlated with green fruit and red ripe fruit yield. Average fruit length Average fruit diameter and average weight of green fruits showed highly significant negative correlation with green fruit and red ripe fruit yield. Fruit length, fruit diameter and average weight of green fruits showed highly significant positive genotypic correlation with green fruit and red ripe fruit yield. Average weight of red ripe fruit, yield, number of seeds/fruit and weight of seeds/fruit showed highly significant positive genotypic correlation with green fruit yield. Ascorbic acid, oleoresin and capsaicin content showed highly significantly positive genotypic correlation with green fruit and red ripe fruit yield.

The nature and magnitude of association between yield and its components traits is necessary for effective selection in advance generations. Dewey and Lu (1959) emphasized that the nature of population under consideration and the magnitude of correlation coefficients could often be influenced by the choice of the individuals upon which the observation are made.
Correlations between characters pairs are due to linkage of genes or pleiotrophy of genes. Therefore, selection of one trait influences the other linked or pleiotrophically affected traits. Selection is usually practiced for changing two or more than two characters simultaneously. The information on interrelationship of the important economic indicated strong genetic associations between the traits and the phenotypic traits may be useful in the prediction of correlated response to direct selection in the construction of some characters which may have no values in themselves but may be useful as indicators of other important traits (Robinson et al., 1951).

Considerable importance has been attached to correlation studies in the plant improvement because they are helpful in making effecting selection. In the present study, correlation between two characters was worked out in all possible combinations at phenotypic and genotypic levels as presented in table ( $3 \& 4)$. In general, the magnitude of genotypic correlation coefficients was higher than the corresponding values of the phenotypic correlation coefficients, the expression which was suppressed due to environmental influence. The present study also suggested that both genotypic and phenotypic correlation were similar in direction. Days to 50 percent flowering was significantly and positively correlated with fruit length, was $s$ harvest and total yield per plant. Fruit length was significantly and positively correlated and with average fruit weight and total yield per plant. The most important trait total yield per plant was significantly and positively correlated with early yield per plant, fruit length per plant. Similar results have been reported by Singh et al., (1972). Sri Latha Kumary and Rajamuny (2002), Nazier et al., (2005) and Choudhar and Samadia (2000). 
Table.1 Phenotypic correlation coefficient for 17 characters in chillies

\begin{tabular}{|c|c|c|c|c|c|c|c|c|c|c|c|c|c|c|c|c|c|}
\hline \multirow[b]{2}{*}{ تج } & \multicolumn{2}{|c|}{ 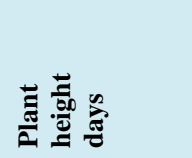 } & \multirow{2}{*}{ 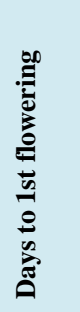 } & \multirow{2}{*}{ 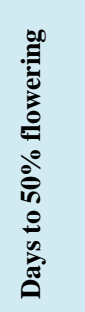 } & \multirow{2}{*}{ 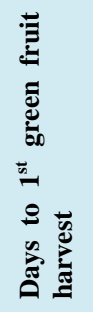 } & \multirow{2}{*}{ 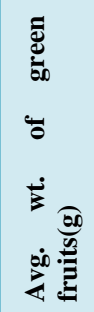 } & \multirow{2}{*}{ 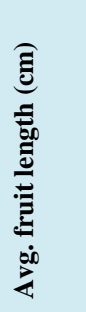 } & \multirow{2}{*}{ 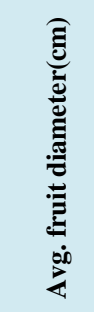 } & \multirow{2}{*}{ 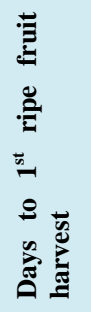 } & \multirow{2}{*}{ 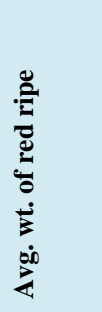 } & \multirow{2}{*}{ 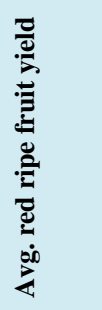 } & \multirow{2}{*}{ 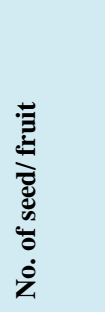 } & \multirow{2}{*}{ 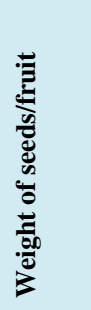 } & \multirow{2}{*}{ 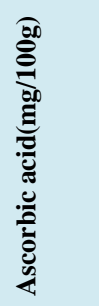 } & \multirow[b]{2}{*}{ 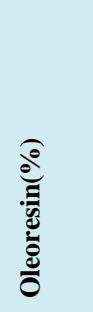 } & \multirow[b]{2}{*}{ 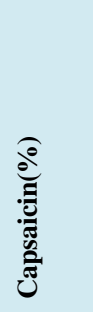 } & \multirow{2}{*}{ 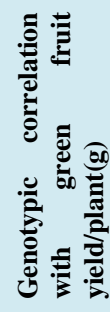 } \\
\hline & 100 & 120 & & & & & & & & & & & & & & & \\
\hline 1 & 0.986 & 0.970 & 0.421 & 0.388 & -0.410 & 0.456 & 0.401 & 0.207 & 0.034 & 0.554 & -0.0054 & 0.102 & 0.034 & 0.051 & 0.488 & -0.548 & 0.308 \\
\hline 2 & & 0.985 & 0.357 & 0.363 & -0.382 & 0.459 & 0.396 & 0.174 & 0.020 & 0.562 & -0.0721 & 0.063 & 0.044 & 0.099 & 0.488 & -0.492 & 0.292 \\
\hline 3 & & & 0.344 & 0.349 & -0.320 & 0.437 & 0.351 & 0.126 & -0.017 & 0.573 & -0.1097 & 0.041 & 0.012 & 0.079 & 0.428 & -0.470 & -0.329 \\
\hline 4 & & & & 0.581 & -0.187 & 0.196 & 0.114 & 0.071 & -0.018 & -0.051 & 0.0190 & 0.215 & 0.035 & -0.0384 & 0.278 & -0.346 & -0.062 \\
\hline 5 & & & & & -0.123 & 0.035 & 0.091 & -0.275 & -0.056 & -0.0162 & -0.105 & -0.016 & 0.077 & -0.270 & 0.164 & -0.320 & -0.062 \\
\hline 6 & & & & & & -0.299 & -0.579 & -0.140 & -0.161 & -0.095 & -0.189 & -0.0205 & -0.046 & -0.467 & -0.102 & 0.336 & 0.183 \\
\hline 7 & & & & & & & 0.264 & 0.461 & 0.127 & 0.208 & 0.185 & 0.489 & 0.0371 & 0.374 & -0.074 & -0.328 & 0.175 \\
\hline 8 & & & & & & & & 0.519 & -0.035 & 0.221 & 0.155 & 0.306 & 0.305 & 0.148 & 0.195 & -0.174 & 0.280 \\
\hline 9 & & & & & & & & & 0.168 & 0.242 & 0.140 & 0.265 & 0.333 & 0.114 & 0.232 & 0.132 & -0.178 \\
\hline 10 & & & & & & & & & & -0.007 & -0.186 & -0.105 & 0.243 & 0.347 & 0.261 & -0.232 & 0.189 \\
\hline 11 & & & & & & & & & & & -0.141 & 0.117 & -0.065 & 0.125 & 0.524 & -0.142 & 0.186 \\
\hline 12 & & & & & & & & & & & & 0.756 & 0.245 & -0.020 & -0.164 & -0.290 & 0.213 \\
\hline 13 & & & & & & & & & & & & & 0.333 & 0.145 & -0.383 & -0.357 & 0.173 \\
\hline 14 & & & & & & & & & & & & & & 0.299 & -0.080 & -0.230 & 0.049 \\
\hline 15 & & & & & & & & & & & & & & & -0.281 & -0.057 & 0.000 \\
\hline 16 & & & & & & & & & & & & & & & & -0.117 & 0.720 \\
\hline 17 & & & & & & & & & & & & & & & & & 0.095 \\
\hline
\end{tabular}

Table.2 Genotypic correlation coefficient for 17 characters in chillies

\begin{tabular}{|c|c|c|c|c|c|c|c|c|c|c|c|c|c|c|c|c|c|}
\hline \multirow[t]{2}{*}{ 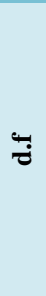 } & \multicolumn{2}{|l|}{ 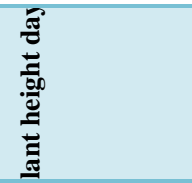 } & \multirow{2}{*}{ 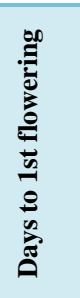 } & \multirow{2}{*}{ 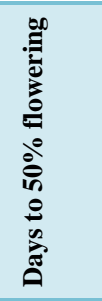 } & \multirow{2}{*}{ 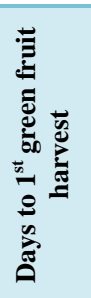 } & \multirow{2}{*}{ 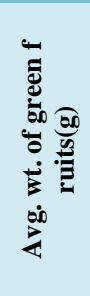 } & \multirow{2}{*}{ 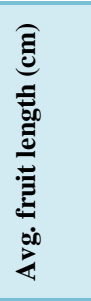 } & \multirow{2}{*}{ 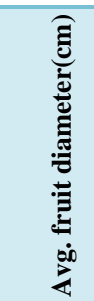 } & \multirow{2}{*}{ 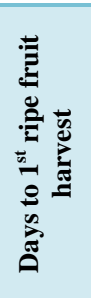 } & \multirow{2}{*}{ 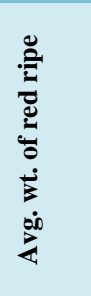 } & \multirow{2}{*}{ 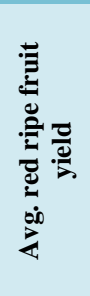 } & \multirow{2}{*}{ 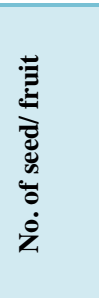 } & \multirow{2}{*}{ 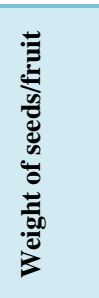 } & \multirow{2}{*}{ 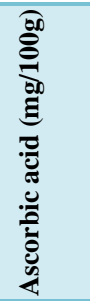 } & \multirow{2}{*}{ 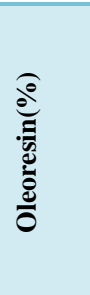 } & \multirow{2}{*}{ 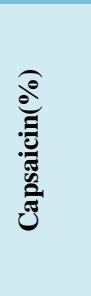 } & \multirow{2}{*}{ 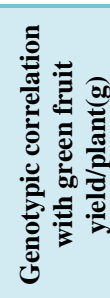 } \\
\hline & 100 & 120 & & & & & & & & & & & & & & & \\
\hline 1 & 0.990 & 0.975 & 0.508 & 0.484 & -0.530 & 0.545 & 0.474 & 0.238 & 0.105 & 0.593 & -0.007 & 0.139 & 0.043 & 0.035 & 0.539 & -0.615 & 0.345 \\
\hline 2 & & 0.992 & 0.426 & 0.456 & -0.504 & 0.546 & 0.464 & 0.195 & 0.096 & 0.600 & -0081 & 0.090 & 0.055 & 0.093 & 0.0498 & -0.556 & 0.345 \\
\hline 3 & & & 0.423 & 0.448 & -0.431 & 0.523 & 0.411 & 0.145 & 0.061 & 0.626 & -0.124 & 0.081 & 0.018 & 0.077 & 0.474 & -0.556 & 0.330 \\
\hline 4 & & & & 0.0642 & -0.268 & 0.217 & 0.148 & 0.047 & -0.043 & -0.045 & 0.205 & 0.0231 & 0.033 & -0.448 & 0.331 & -0.532 & -0372 \\
\hline 5 & & & & & -0.183 & 0.037 & 0.111 & -0.332 & -0.045 & 0.151 & -0.126 & -0.035 & 0.085 & -0.337 & 0.176 & -0.372 & -0.067 \\
\hline 6 & & & & & & -0.317 & -0.630 & -0.189 & -0.192 & -0.095 & -0.215 & -0.228 & -0.051 & -0.517 & -0.110 & 0.354 & 0.163 \\
\hline 7 & & & & & & & 0.271 & 0.497 & 0.162 & 0.213 & 0.188 & 0.524 & 0.379 & 0.386 & -0.082 & -0.331 & 0.196 \\
\hline 8 & & & & & & & & 0.601 & -0.020 & 0.235 & 0.157 & 0.318 & 0.315 & 0.171 & 0.200 & -0.182 & 0.181 \\
\hline 9 & & & & & & & & & 0.150 & 0.247 & 0.147 & 0.299 & 0.363 & 0.095 & 0.254 & -0.139 & -0.283 \\
\hline 10 & & & & & & & & & & 0.025 & -0.227 & -0.178 & 0.271 & 0.402 & 0.298 & -0.264 & 0.113 \\
\hline 11 & & & & & & & & & & & -0.143 & -0.116 & -0.066 & 0.118 & 0.546 & -0.144 & 0.195 \\
\hline 12 & & & & & & & & & & & & 0.788 & 0.247 & -0.019 & -0166 & -0.294 & 0.186 \\
\hline 13 & & & & & & & & & & & & & 0.345 & 0.153 & -0.399 & -0375 & 0.223 \\
\hline 14 & & & & & & & & & & & & & & 0.311 & -0.079 & -0.231 & 0.172 \\
\hline 15 & & & & & & & & & & & & & & & -0.298 & -0.061 & 0.048 \\
\hline 16 & & & & & & & & & & & & & & & & -0.117 & 0.001 \\
\hline 17 & & & & & & & & & & & & & & & & & 0.191 \\
\hline
\end{tabular}


Table.3 Phenotypic direct (diagonal) and indirect effects of 17 characters in chillies

\begin{tabular}{|c|c|c|c|c|c|c|c|c|c|c|c|c|c|c|c|c|c|}
\hline \multirow[b]{2}{*}{$\underset{\dot{n}}{\dot{0}}$} & \multicolumn{2}{|c|}{ 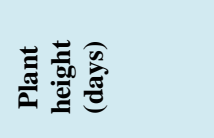 } & \multirow{2}{*}{ 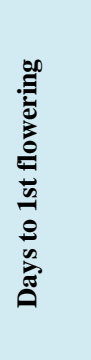 } & \multirow{2}{*}{ 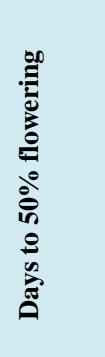 } & \multirow{2}{*}{ 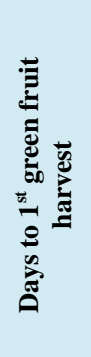 } & \multirow{2}{*}{ 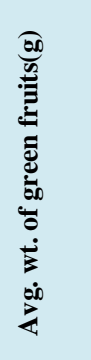 } & \multirow{2}{*}{ 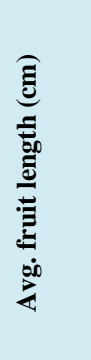 } & \multirow{2}{*}{ 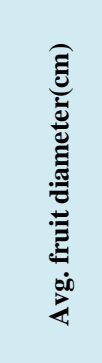 } & \multirow{2}{*}{ 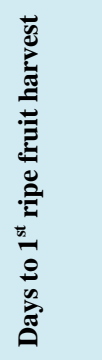 } & \multirow{2}{*}{ 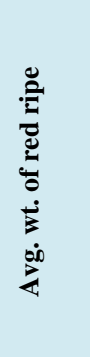 } & \multirow{2}{*}{ 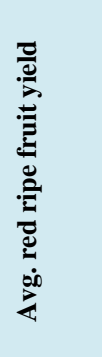 } & \multirow{2}{*}{ 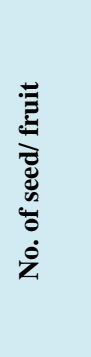 } & \multirow{2}{*}{ 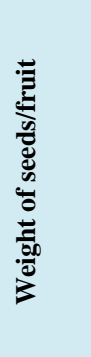 } & \multirow{2}{*}{ 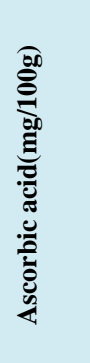 } & \multirow{2}{*}{ 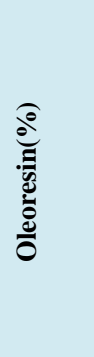 } & \multirow{2}{*}{ 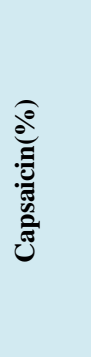 } & \multirow{2}{*}{ (0) } \\
\hline & 100 & 120 & & & & & & & & & & & & & & & \\
\hline 1 & -0.691 & -0.680 & -0.295 & 0.272 & 0.287 & -0.319 & -0.281 & -0.145 & -0.023 & -0.388 & -0.038 & -0.072 & -0.024 & -0.036 & -0.342 & -0.548 & 0.216 \\
\hline 2 & 0.077 & 0.076 & 0.027 & 0.028 & -0.029 & 0.035 & 0.030 & 0.013 & 0.001 & 0.043 & -0.005 & 0.004 & 0.003 & 0.007 & 0.034 & -0.492 & 0.002 \\
\hline 3 & 0.470 & 0.476 & 0.164 & 0.166 & -0.153 & 0.208 & 0.167 & 0.060 & 0.008 & 0.273 & -0.052 & 0.020 & 0.005 & 0.037 & 0.204 & -0.470 & -0.157 \\
\hline 4 & -0.037 & -0.036 & -0.105 & -0.061 & 0.019 & -0.020 & -0.012 & -0.007 & 0.002 & 0.005 & -0.020 & -0.022 & -0.003 & 0.040 & -0.029 & -0.346 & -0.006 \\
\hline 5 & -0.043 & -0.041 & -0.069 & -0.119 & -0.014 & -0.004 & -0.010 & 0.032 & 0.006 & 0019 & 0.012 & 0.002 & -0.009 & 0.032 & -0.019 & -0.320 & -0.017 \\
\hline 6 & -20.125 & -0.104 & -0.061 & -0.040 & -0.327 & -0.097 & -0.189 & -0.046 & -0.052 & 0.031 & -0.061 & -0.067 & -0.015 & -0.152 & 0.033 & 0.366 & 0.060 \\
\hline 7 & 0.058 & 0.055 & 0.024 & 0.004 & -0.038 & 0.127 & 0.033 & 0.058 & 0.016 & 0.026 & 0.023 & 0.062 & 0.047 & 0.047 & -0.009 & -0.328 & 0.022 \\
\hline 8 & -0.038 & -0.034 & -0.011 & -0.008 & 0.056 & -0.025 & -0.096 & -0.050 & 0.003 & -0.021 & -0.015 & -0.029 & -0.029 & -0.014 & -0.018 & -0.174 & 0.027 \\
\hline 9 & -0.046 & -0.033 & -0.018 & 0.073 & 0.073 & -0.122 & -0.137 & -0.0265 & -0.044 & -0.064 & -0.037 & -0.070 & -0.088 & -0.030 & -0.061 & -0.132 & 0.047 \\
\hline 10 & -0.241 & -0.272 & -0.051 & -0.118 & -0.152 & -0.144 & 0.231 & 0.147 & -0.079 & -0.156 & -0.153 & -0.176 & -0.143 & 0.040 & 0.000 & 0.720 & 0.827 \\
\hline 11 & -0.002 & 0.002 & 0.002 & 0.007 & 0.021 & -0.017 & 0.004 & -0.002 & -0.134 & 0.001 & 0.025 & 0.014 & -0.032 & -0.046 & -0.035 & -0.033 & 0.128 \\
\hline 12 & 0.025 & 0.025 & -0.002 & -0.007 & -0.004 & 0.009 & 0.009 & 0.010 & -0.0003 & 0.044 & -0.006 & -0.005 & -0.002 & 0.005 & 0.023 & -0.142 & 0.008 \\
\hline 13 & 0.001 & 0.002 & -0.004 & 0.002 & 0.004 & -0.004 & -0.003 & -0.003 & 0.004 & 0.003 & -0.023 & -0.017 & -0.005 & 0.005 & 0.023 & -0.142 & 0.004 \\
\hline 14 & 0.003 & 0.002 & 0.011 & -0.0009 & -0.011 & 0.027 & 0.016 & 0.014 & -0.005 & -0.006 & 0.041 & -0.055 & 0.018 & 0.008 & -0.021 & -0.357 & 0.011 \\
\hline 15 & 0.002 & 0.006 & 0.001 & 0.004 & -0.002 & 0.019 & 0.016 & 0.017 & 0.012 & -0.003 & 0.013 & 0.017 & 0.052 & 0.015 & -0.004 & -0230 & 0.009 \\
\hline 16 & 0.004 & 0.003 & 0.017 & -0.012 & -0.020 & 0.016 & 0.006 & 0.005 & 0.015 & 0.005 & -0.0009 & -0.006 & 0.013 & 0.044 & -0.012 & -0.057 & 0.002 \\
\hline 17 & 0.091 & 0.087 & 0.056 & 0.033 & -0.020 & -0.015 & 0.039 & 0.047 & 0.053 & 0.107 & -0.033 & -0.078 & -0.016 & -0.057 & -0.204 & -0.204 & 0.000 \\
\hline
\end{tabular}

Table.4 Genotypic direct (diagonal) and indirect effects of 17 characters in chillies

\begin{tabular}{|c|c|c|c|c|c|c|c|c|c|c|c|c|c|c|c|c|c|}
\hline \multirow{2}{*}{$\underset{\dot{m}}{\dot{\phi}}$} & \multicolumn{2}{|c|}{ 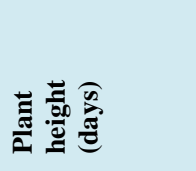 } & \multirow{2}{*}{ 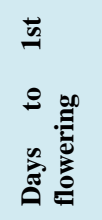 } & \multirow{2}{*}{ 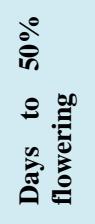 } & \multirow{2}{*}{ 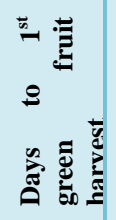 } & \multirow{2}{*}{ 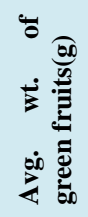 } & \multirow{2}{*}{ 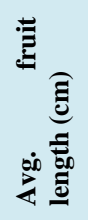 } & \multirow{2}{*}{ 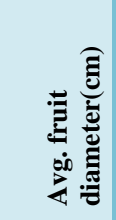 } & \multirow{2}{*}{ 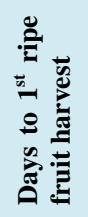 } & \multirow{2}{*}{ 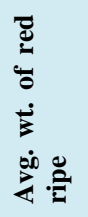 } & \multirow{2}{*}{ 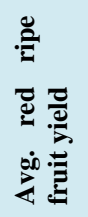 } & \multirow{2}{*}{$\begin{array}{l}\vec{\Phi} \\
\dot{w} \\
\ddot{\sigma} \\
\dot{z}\end{array}$} & \multirow[t]{2}{*}{ 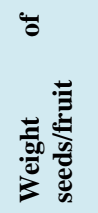 } & \multirow[t]{2}{*}{ 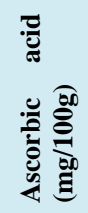 } & \multirow{2}{*}{ 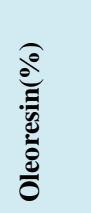 } & \multirow{2}{*}{ 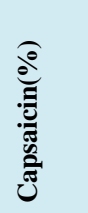 } & \multirow[t]{2}{*}{ 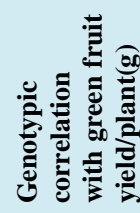 } \\
\hline & 100 & 120 & & & & & & & & & & & & & & & \\
\hline 1 & 3.96 & 3.90 & 2.034 & 1.939 & -2.123 & 2.181 & 1.898 & 952 & 0.423 & 2.376 & -0.615 & -0.031 & 0.557 & 0.175 & 0.142 & 2.16 & 1.382 \\
\hline 2 & & 8830 & & -4.063 & 4.497 & -4.866 & -4.139 & 170 & 2.944 & -0.858 & -5.350 & 0.72 & 0805 & -0.497 & -0.8 & -4.4 & 0. \\
\hline 3 & 358 & 2.376 & 1 & 1066 & -1.024 & 1.242 & 0.977 & 0.532 & -0.884 & 0.147 & 1.487 & -0.295 & 0.192 & 0.044 & 0.18 & 1.12 & 0.344 \\
\hline 4 & -1 & -0.0 & -0 & -0.074 & 0.031 & -0.025 & -0.017 & 0.00 & 0.0 & .005 & 0.005 & -0.023 & -0.026 & -0.003 & 0.1 & 0 & 0.38 \\
\hline 5 & .515 & -0.507 & -0.7 & -1.129 & 0.207 & -0.042 & -0.125 & 0.375 & 0.18 & 0.051 & 0.170 & 0.143 & 0.040 & -0.096 & 0.3 & 8 & -0.3 \\
\hline 6 & 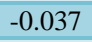 & -0.031 & -0.0 & -0.013 & 0.073 & -0.023 & -0.046 & -0.014 & -0. & -0.014 & -0.007 & -0.015 & -0.016 & -0.003 & 0 & -0 . & -0.3 \\
\hline 7 & 428 & 1.36 & 0. & 0.097 & -0.830 & 2.614 & 0.708 & 1.299 & -0.47 & 0.424 & 0.556 & 0.491 & 1.370 & 0.992 & 1.0 & -0.214 & 0.33 \\
\hline 8 & 904 & 0.853 & & 0.230 & -1.309 & 0.563 & 2.076 & 248 & 0.588 & -0.043 & 0.488 & 0.326 & 0.661 & 0.655 & 0.35 & 0.415 & 0.182 \\
\hline 9 & 526 & -0390 & 0 & 0802 & 0510 & $-1,336$ & 1 & 688 & -0.5 & -0.403 & -0.665 & -0.396 & 806 & -0 & & 0.684 & 0.1 \\
\hline 10 & 043 & 0.048 & 0 & 021 & 0.025 & 0.0 & -0.036 & -0.02 & -0.1 & 0.0 & 0.025 & 0.024 & 0.029 & 0.022 & 0. & 0.0 & 0.723 \\
\hline 11 & & & & & & & 0.01 & & 01 & & & & & & & & 0.264 \\
\hline 12 & $-0.2 \varepsilon$ & -0 & & & & & 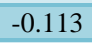 & & 0.0 & & -0. & 0. & 0.0 & & & 63 & 0.144 \\
\hline 13 & 0.0 & 0.1 & & 0.1 & 0.2 & & -0 . & & 0.2 & 0.2 & 01 & -1.110 & 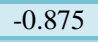 & -0 & & & 0.294 \\
\hline 14 & & & & & & & & & & & & & & & & & 0.375 \\
\hline 15 & 0.010 & 0.003 & 0 & 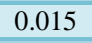 & -0.009 & 0.070 & 0.05 & 0.067 & -0.1 & 0.0 & -0.012 & 0.0 & 0.063 & 0.1 & 0.0 & -0.014 & 0.231 \\
\hline 16 & -0.007 & -0.005 & 0.033 & 0.025 & 0.038 & -0.028 & -0.012 & -0.007 & -0.003 & -0.030 & -0.008 & 0.001 & -0.011 & -0.023 & -0.074 & 0.022 & 0.061 \\
\hline 17 & 1.018 & 0.970 & 0.636 & 0.359 & -0.226 & -0.168 & 0.409 & 0.520 & -0.002 & 0.610 & 1.117 & -0.340 & -0.817 & -0.162 & -0.609 & 2.044 & 0.117 \\
\hline
\end{tabular}




\section{Path coefficient analysis}

The data were subjected to path coefficient analysis to partition the correlation coefficients of all the component characters with green fruit yield per plant in to direct and indirect effects. The results of various causes at genotypic and phenotypic level influencing average fruit weight per plant (effect) are presented in table 2.1 and table 2.2. The path coefficient analysis revealed that the characters like to first flowering (0.027), days to $50 \%$ flowering $(0.166)$, days to $1^{\text {st }}$ green fruit harvest $(0.019)$ and fruit diameter $(0.058)$ exercised maximum direct effect on fruit yield at phenotypic level. High positive direct effect of number of seeds (0.91), ascorbic acid (9.005) and $1^{\text {st }}$ ripe fruit harvest (0.003) and fruit diameter was the main cause of their positive association with fruit yield. Plant height (-0.680), weight of green fruits ($0.004)$, fruit length $(-0.189)$ and weight of red ripe (-0.064) exercised minimum direct effect on fruit yield phenotypic level, high negative direct effect of weight of seeds/fruit (-0.002), oleoresin a(-0.021) and capsaicin (-0.230) was the main cause of their negative association with average red ripe fruit yield. All these characters had positive and negative direct and indirect effect on total yield per plant height (3.902), days to 50\% flower (1.066), days to $1^{\text {st }}$ green fruit harvest, fruit diameter (1.299), days to $1^{\text {st }}$ ripe fruit harvest $(0.588)$. Red ripe fruit yield $(0.25)$,number of seeds/fruit (0.206), weight of seeds/fruit (0.056) exercise maximum direct effect on fruit yield at genotypic level. Days to $1^{\text {st }}$ flowering (-3.797), weight of green fruit ($0.403)$, ascorbic acid (-0.274) oleoresin ($0.023)$ and capsaicin content $(-0.014)$ had minimum negative direct effect on fruit yield. The estimates of residual effect reflect the adequacy and appropriateness of the character chosen for path coefficient analysis. In the present investigation the residual effect was very high (1.729) indicating the adequacy of the characters chosen for the study and the characters studied contributed approximately 90 percent towards yield. Correlation measures only mutual relationship with concern to cause and effect. Path coefficient analysis provides a method for separating out direct and indirect effects and measures the relative importance of causal factors which ultimately affect the yield. Path coefficient is simply standardized partial regression coefficient which splits the correlation coefficients into the measures of the direct and indirect effects of a set of independent variable on the dependent variable. The analysis of path coefficient revealed highest amount of positive direct effect for yield per plant followed by fruit weight, days to 50 percent flowering and plant height has also positive direct effect on yield at genotypic and phenotypic level. However, fruit length per plant, ascorbic acid, oleoresin and capsaicin content had negative direct effect on yield at both genotypic and phenotypic levels. Fruit length and diameter of fruit had negative direct effect yield at genotypic level. Similar results were also reported by Nanda Devi et al., (2003), Nazier et al., (2005), and Devi and Arumugam (1999) that green fruit yield per plant had the positive effect on dry fruit yield per plant.

In conclusion, the magnitude of genotypic correlation was higher than the phenotypic correlation for all the character. The most important trait green fruit yield per plant was significant and positively correlated with phenotypic correlation average red ripe fruit yield plant height days to $1^{\text {st }}$ ripe fruit harvest, length and fruit diameter, weight of red ripe fruit, no of seeds/fruit, weight of seeds/fruit, ascorbic acid oleoresin and capsaicin content. Mostly important trait of green fruit yield per plant was significantly and negative correlated with phenotypic correlation with days to first flowering, days to $50 \%$ flowering, days to first green fruit harvest and 
days to first ripe fruit harvest. The most important traits green fruit yield was significantly and positive correlated with genotypic correlation with plant height, average weight of green fruits, average fruit length average, fruit diameter, average weight of red ripe, average red ripe fruit yield, No. of seeds/fruit, weight of seeds, Ascorbic acid, oleoresin and capsaicin. Mostly important trait green fruit yield was significantly and negative correlated with genotypic correlation with days to first flowering, days to $50 \%$ flowering, days to first green fruit harvest, and days to first fruit harvest. Path analysis indicated positive direct contribution of days to first flowering, days to $50 \%$ flowering, Average fruit diameter days to first ripe fruit harvest, No. of seeds/fruit, Ascorbic acid and green fruit with phenotypic levels and phenotypic levels. Plant height, weight of green fruit, Average weight of green fruit, Average weight of red ripe fruit fruits length, weight of seeds, oleoresin, Ascorbic acid and capsaicin content had negative indirect effect at phenotypic levels. Plant height, days to $50 \%$ flowering, days to first green fruit harvest, fruit diameter, days to first harvest , Average red ripe fruit yield, No of seeds/fruit, weight of seeds/fruit, with green fruit yield had positive direct effect genotypic levels. Path analysis indicated negative indirect contribution of days to first flowering, Average weight of green fruit, Average fruit length, Average weight of red ripe fruit, Ascorbic acid, Oleoresin and capsaicin with genotypic levels. Based on per se performance SM-20, JCA-9, Pusa Jawaala, Utkal Ragini and Kashi anmol exhibited high fruit yield per plant. SM-20 found to be highest yield i.e. $168.42 \mathrm{~g} /$ plant.

\section{References}

Al-Jibouri, H.A.; Miller, P.A and Robinson, H.F. (1958). Genetic and environmental variance and covariance in an upland cotton cross of interspecific origin. Agron. J.,50: 633-636.

Choudhary, B.S and Samodia, D.K. (2004). "Variability and character association in chilli land racess and genotypes under arid environment. India. J.Hort. 61(2) 132-136.

Devi, D.S and Arumugam (1999). "Correlation and Path coefficient analysis." Res. Hisar, 17(1): 90-93.

Deway, D.R and L, K.H. (1959). Corellation and Path coefficient analysis of component of crested wheat grass seed production. Indian J. Agron., 51: 515518.

Lenka, D. and Mishra, B. (1973). Path coefficient analysis of yield in rice varieties. Indian J. Agric. Science 43: 376-379.

Nandadevi and Hosamani, R.(2003). Variability, correlation and path analysis in Kharif grown chilli (capsicum annuum L.)". Capsicum and Eggplant News letter, (22): 43-46.

Nazier, G. Narayan, R. , Hussian, K. Ahmed, N. and Bhat, K.(2005) " Correlation and Path coefficient analysis in sweet papper Veg. Sci. 3(1) 88-89.

Robinson, H.F.; Comstock, R.E and Harvery, P.H.(1951). "Genotypic and Phenotypic correlation in corn and their implication in selection." Agron. J., 43: 282-287.

Singh, H.N., Singh, B., Kallo, G. and Mehrotra, N.(1972). Genetic variability and correlation studies in (Capsicum annuum L.). Haryana J. Agric. Res., 11(1): 13-18.

Sreelatha Kumary and Rajamony (2002). "Pheneotypic and genotypic coefficient in Capsicum annuum L" .Indian J. of Hort. 59(1):77-83. 


\section{How to cite this article:}

Mishra, T.S. and Singh, H.M. 2020. Study on Phenotypic, Genotypic Correlation and Path Coefficients in Chilli. Int.J.Curr.Microbiol.App.Sci. 9(07): 1930-1937. doi: https://doi.org/10.20546/ijcmas.2020.907.220 